\title{
Raquianestesia com Bupivacaína a 0,5\% Isobárica para Cirurgia Pediátrica Ambulatorial em Pacientes com Idades de 6 a 12 Anos. Estudo Prospectivo *
}

\section{Isobaric 0.5\% Bupivacaine for Spinal Anesthesia in Pediatric Outpatient Surgery of 6 to 12 Year Old Children. A Prospective Study}

Luiz Eduardo Imbelloni, TSA ${ }^{1}$, Eneida Maria Vieira ${ }^{2}$, Lúcia Beato, TSA ${ }^{1}$, Carlos Zapatta ${ }^{3}$

\begin{abstract}
RESUMO
Imbelloni LE, Vieira EM, Beato L, Zapatta C - Raquianestesia com Bupivacaína a $0,5 \%$ Isobárica para Cirurgia Pediátrica Ambulatorial em Pacientes com Idades de 6 a 12 Anos. Estudo Prospectivo
\end{abstract}

Justificativa e Objetivos - Em crianças, a raquianestesia produz profunda analgesia e, quando combinada com anestesia geral, reduz a necessidade dos agentes anestésicos e opióides. O objetivo deste estudo prospectivo foi avaliar as características clínicas da raquianestesia com bupivacaína a $0,5 \%$ racêmica em 40 crianças, com idades entre 6 e 12 anos.

Método - Participaram do estudo prospectivo 40 crianças com idades entre 6 e 12 anos, submetidas à raquianestesia com bupivacaína a 0,5\% isobárica, na dose de $0,5 \mathrm{mg}^{\mathrm{kg}}{ }^{-1}$. Foram avaliados os seguintes parâmetros: latência da analgesia, bloqueio motor, duração dos efeitos, dispersão cranial da analgesia, alterações cardiovasculares, cefaléia, sintomas neurológicos transitórios.

Resultados - O tempo de latência foi de $2,60 \pm 1,28$ minutos. A duração da analgesia foi de 4,51 $\pm 0,89 \mathrm{~h}$. O tempo para a deambulação foi de 4,04 $\pm 0,83 \mathrm{~h}$. O tempo de permanência na SRPA foi de 44,39 $+43,13$ minutos. A duração do bloqueio motor foi de 2,50 $\pm 0,83 \mathrm{~h}$. O nível sensitivo do bloqueio variou entre $T_{9}$ e $T_{4}\left(\right.$ Moda $=T_{6}$ ). O início do bloqueio motor foi menor que três minutos em todas as crianças. Logo no início da cirurgia, todos os pacientes apresentaram bloqueio motor grau 3 (escala modificada de Bromage). Setenta porcento dos pacientes apresentaram bloqueio motorgraus 3 ou 2 no final da cirurgia. Não foi observada dessaturação ou hipotensão arterial. Bradicardia foi observada em dois pacientes. Ocorreu uma falha. Cefaléia e SNT não foram observadas.

Conclusões - Nas condições deste estudo a bupivacaína a $0,5 \%$ isobárica produziu anestesia segura em pacientes de 6 a 12 anos em regime ambulatorial, com alto índice de sucesso, bloqueio motor de duração intermediária e baixa incidência de efeitos colaterais. Os resultados mostraram que a

\footnotetext{
* Recebido do (Received from) CET/SBA do Hospital de Base da Faculdade de Medicina de São José do Rio Preto (FAMERP) e Clínica São Bernardo, Rio de Janeiro. $R J$

1. Anestesiologista da Casa de Saúde Santa Maria e Clínica São Bernardo, Rio de Janeiro, RJ

2. Auxiliar de Ensino do Hospital de Base da FAMERP

3. $M E_{2}$ do CET/SBA do Hospital de Base da FAMERP
}

Apresentado (Submitted) em 09 de outubro de 2001

Aceito (Accepted) para publicação em 03 de janeiro de 2002

Correspondência para (Mail to)

Dr. Luiz Eduardo Imbelloni

Av. Epitácio Pessoa, 2356/203-Lagoa

22471-000 Rio de Janeiro, $R J$

E-mail: imbelloni@openlink.com.br

(C) Sociedade Brasileira de Anestesiologia, 2002 raquianestesia é segura e facilmente realizável em crianças de 6 a 12 anos em regime ambulatorial.

UNITERMOS - ANESTESIA, Ambulatorial; ANESTÉSICOS, Local: bupivacaína; CIRURGIA, Pediátrica; TÉCNICAS ANESTÉSICAS, Regional: subaracnóidea

\section{SUMMARY}

Imbelloni LE, Vieira EM, Beato L, Zapatta C - Isobaric 0.5\% Bupivacaine for Spinal Anesthesia in Pediatric Outpatient Surgery of 6 to 12 Year Old Children. A Prospective Study

Background and Objectives - Spinal anesthesia produces deep intra-operative analgesia in children and, when combined with general anesthesia, decreases perioperative anesthetics and opioids requirements. This prospective study aimed at evaluating clinical characteristics of spinal anesthesia with $0.5 \%$ racemic bupivacaine in 40 children, aged 6 to 12 years.

Methods - Participated in this prospective study, 40 patients aged 6 to 12 years submitted to spinal anesthesia with 0.5 $\mathrm{mg} . \mathrm{kg}^{-1}$ of $0.5 \%$ plain bupivacaine. The following parameters were evaluated: onset of analgesia, motor block, duration of effects, level of cephalad spread, cardiovascular changes, headache and transient neurological symptoms.

Results - Onset time was $2.60 \pm 1.28 \mathrm{~min}$. Analgesia duration was $4.51 \pm 0.89 \mathrm{~h}$. Time for ambulation was $4.04 \pm 0.83 \mathrm{~h}$. PACU stay was $44.39 \pm 43.13 \mathrm{~min}$. Motor block duration was $2.50 \pm$ $0.83 \mathrm{~h}$. Sensory block level varied from $T_{9}$ to $T_{4}\left(\right.$ Mode $\left.=T_{6}\right)$. Motor block onset was less than three minutes in all children. All of them presented motor block grade 3 (modified Bromage Score) at the beginning of the surgery. Over $70 \%$ of all patients recovered to motor block 3 or 2 at surgery completion. No patient showed oxygen desaturation or arterial hypotension. Bradycardia was observed in two patients. There has been one motor block failure. There were no PDPH or transient neurological symptoms.

Conclusions - In our study, 0.5\% isobaric bupivacaine induced a safe anesthesia in patients aged 6 to 12 years in outpatient procedures, with a high success rate, short-lived motor block and a low incidence of side effects. Our results have shown that spinal anesthesia is a safe and very easy technique for children between 6 and 12 years of age for outpatient procedures.

KEY WORDS: ANESTHESIA, Outpatient procedure; ANESTHETICS, Local: bupivacaine; ANESTHETIC TECHNIQUES, Regional: spinal block; SURGERY, Pediatric

\section{INTRODUÇÃO}

primeiro relato de raquianestesia em criança data de $1901^{1}$. A técnica não obteve grande popularidade até $1980^{2,3}$, quando foi reintroduzida como alternativa da anestesia geral em recém-nascidos de alto risco ${ }^{4-6}$ e se tornou popular para crianças de 2 meses a 12 anos ${ }^{5-7}$. No Hospital 
de Base a raquianestesia não era utilizada até maio de 2001, quando passou a ser rotina do serviço para cirurgias ambulatoriais de crianças de 1 a 5 anos $^{8}$. Ela produz rápido início de ação, com uniforme distribuição de analgesia e excelente relaxamento muscular.

Os anestésicos locais do tipo amida (bupivacaína e lidocaína) são usados regularmente para raquianestesia em crianças com pequenas doses e baixo risco de toxicidade sistêmica. A duração de ação da lidocaína pode ser curta para algumas crianças submetidas à cirurgias em regime ambulatorial ${ }^{3,9}$. A bupivacaína apresenta uma duração de ação maior do que a lidocaína.

A baricidade do anestésico local é um dos mais importantes fatores que influencia a distribuição do anestésico dentro do $\operatorname{LCR}^{10}$. Soluções hiperbáricas ${ }^{2,3,11}$ e isobáricas ${ }^{8,11}$ são utilizadas em raquianestesia de crianças com excelentes resultados.

O objetivo deste estudo foi avaliar as características clínicas da bupivacaína a $0,5 \%$ isobárica em raquianestesia para cirurgias pediátricas. Foram avaliadas a dispersão e a regressão da analgesia, o tempo de alta da SRPAe a alta hospitalar.

\section{MÉTODO}

Durante dois meses, 40 pacientes escalados para cirurgias pediátricas em regime ambulatorial com idades entre 6 e 12 anos sob raquianestesia com bupivacaína a $0,5 \%$ na dose de $0,5 \mathrm{mg} \cdot \mathrm{kg}^{-1}$ foram incluídos neste estudo após aprovação das Comissões de Ética dos Hospitais (Tabela I). Aos pais e às crianças maiores foi explicado o estudo após o que eles deram seu consentimento. Pacientes com contra-indicação para raquianestesia, como aumento da pressão intracraniana, distúrbios hemorrágicos, distúrbios neurológicos ou infecção no local de punção foram excluídos do estudo.

Tabela I - Tipos de Cirurgias

\begin{tabular}{ll}
\hline Criptorquidia & 1 \\
Fístula ureteral & 2 \\
Herniorrafia inguinal unilateral & 9 \\
Herniorrafia inguinal bilateral & 7 \\
Herniorrafia inguinal + Postectomia & 2 \\
Herniorrafia umbilical + Inguinal & 2 \\
Herniorrafia inguinal + Orquidopexia & 1 \\
Herniorrafia inguinal + Hidrocelectomia & 1 \\
Hidrocelectomia & 2 \\
Hipospádia & 1 \\
Orquidopexia & 4 \\
Postectomia & 6 \\
Postectomia + Hidrocelectomia & 2 \\
\hline
\end{tabular}

As crianças foram instruídas para não se alimentarem com produtos sólidos ou leite durante 6 a 8 horas que antecedem a anestesia. Informações a respeito do jejum também foram dadas aos pais. Líquidos sem resíduos puderam ser ingeri- dos até duas horas antes da indução da anestesia. Cada criança recebeu midazolam $\left(0,8 \mathrm{mg} \cdot \mathrm{kg}^{-1}\right)$ por via oral como medicação pré-anestésica, 20 minutos antes da entrada na SO. Aindução anestésica foi realizada sob máscara com oxigênio e sevoflurano até $5 \%$, seguida de punção venosa e infusão de solução de cloreto de sódio a 0,9\%. Em plano anestésico, as crianças foram colocadas em decúbito lateral esquerdo e realizada a punção lombar nos espaços $L_{3}-L_{4}$ ou $L_{4}-L_{5}$, com agulhas descartáveis pediátricas (26G Atraucan ${ }^{\circledR}$ B.Braun Melsungen AG), sem introdutor, pela via paramediana. Acorreta posição da agulha foi confirmada pela presença do LCR. O bisel foi orientado em sentido cefálico e a dose calculada de bupivacaína a $0,5 \%$ isobárica em embalagem esterilizada foi injetada, sem barbotagem, na velocidade de 1 $\mathrm{ml} .15 \mathrm{~s}^{-1}$ e os pacientes colocados imediatamente em posição supina horizontal.

Amonitorização per-operatória consistiu de medida da pressão arterial por método não invasivo a cada 5 minutos, da freqüência cardíaca e da oximetria de pulso $\left(\mathrm{SpO}_{2}\right)$. O ECG foi monitorizado continuamente na posição CM5. Quando ocorria diminuição de mais de $30 \%$ dos níveis da pressão arterial verificada na enfermaria, o tratamento inicial foi feito com aumento da infusão de cristalóides e posteriormente com o uso de efedrina. Afreqüência cardíaca foi considerada normal se estivesse em torno de $100 \mathrm{bpm}$. Se ocorresse diminuição de $15 \%$ de seus valores, seria administrada atropina na dose de $0,01 \mathrm{mg} \cdot \mathrm{kg}^{-1}$. Os valores da PAS, PAD, PAM e FC foram analisados e comparados nos seguintes momentos: chegada à sala de operações (SO), pós-punção raquidiana (PPR), incisão cirúrgica (IC) e término da cirurgia (TC).

Após a realização do bloqueio e descontinuação do sevoflurano, foi pesquisada a latência do bloqueio definida como o tempo para primeira perda de sensibilidade na região correspondente ao metâmero da punção. O nível segmentar da analgesia foi definido como a perda da sensação à picada de agulha e foi testado 10 minutos após a injeção do anestésico. O bloqueio motor avaliado pela escala modificada de Bromage $(0=$ sem bloqueio motor 3 = bloqueio motor completo $)$ no início e final da cirurgia.

Após a cirurgia, as crianças foram transferidas para a SRPA para monitorização contínua dos sinais vitais e verificação da regressão do bloqueio. A duração da analgesia foi considerada quando houve retorno da sensibilidade ao dermátomo correspondente à punção e a duração do bloqueio motor como o retorno completo da atividade muscular nos membros inferiores. Todos os efeitos adversos foram anotados. Em caso de dor foi administrada dipirona na dose de 20 $\mathrm{mg} . \mathrm{kg}^{-1}$ por via venosa. Assim que as crianças moviam livremente os membros inferiores foram transferidas para a unidade de pacientes externos.

A alta hospitalar foi dada quando as crianças estavam acordadas, deambulando normalmente, sinais vitais estáveis, sem dor, sem náuseas ou vômitos e eram capazes de tolerar líquidos e urinar espontaneamente. Os pacientes foram acompanhados até o terceiro dia de pós-operatório para a pesquisa de cefaléia pós-punção ou sintomas neurológicos transitórios (SNT) definidos por dor ou disestesia nas costas, 
nádegas e pernas irradiando para extremidades inferiores e resolução dentro de 72 horas e até o $30^{\circ}$ dia com relação às complicações neurológicas definitivas.

Os resultados estão apresentados em média e desvio padrão e analisados pelos testes Kruskal-Wallis e Wilcoxon Signed Rank (teste da mediana) para comparação dos valores de pressão arterial, freqüência cardíaca e saturação de oxigênio, sendo considerado significativo o valor de $p<0,05$.

\section{RESULTADOS}

Os dados demográficos estão na tabela II.

Tabela II - Dados Demográficos

\begin{tabular}{lc}
\hline Idade $(\mathrm{anos}){ }^{*}$ & $8,15 \pm 1,95$ \\
Peso $(\mathrm{kg})^{*}$ & $31,98 \pm 11,05$ \\
Altura $(\mathrm{cm}){ }^{*}$ & $126,65 \pm 14,12$ \\
Sexo & \\
$\quad$ Masculino & 29 \\
$\quad$ Feminino & 11 \\
\hline
\end{tabular}

*Valores expressos pela Média \pm DP

As características do bloqueio estão na tabela III. A latência foi de $2,60 \pm 2,33$ minutos. A duração da analgesia foi de $4,51 \pm 0,89$ horas. O tempo de deambulação foi de 4,04 \pm 0,61 horas. O tempo de permanência na SRPA foi de $44,39 \pm$ 43,13 minutos. Aduração do bloqueio motor foi de $2,50 \pm 0,83$ horas.

Tabela III - Características do Bloqueio (Média \pm DP)

\begin{tabular}{lc}
\hline Latência $(\mathrm{min})$ & $2,60 \pm 1,28$ \\
Analgesia (h) & $4,51 \pm 0,89$ \\
Duração do bloqueio motor (h) & $2,50 \pm 0,83$ \\
Tempo raquianestesia-deambulação (h) & $4,04 \pm 0,61$ \\
Termo de permanência na SRPA (min) & $44,39 \pm 43,13$ \\
\hline
\end{tabular}

O nível superior da analgesia variou de $\mathrm{T}_{9}$ a $\mathrm{T}_{4} \mathrm{com}$ a Moda em $\mathrm{T}_{6}$ (Figura 1).

O tempo para início do bloqueio motor foi menor do que três minutos em todas as crianças. Todos apresentaram bloqueio motor grau 3 no início da cirurgia. No final, foi observado bloqueio motor grau 3 em 15 pacientes, grau 2 em 13, grau 1 em 9 , e zero em 3 . Setenta por cento dos pacientes apresentaram bloqueio motor 3 ou 2 no final da cirurgia (Figura 2).

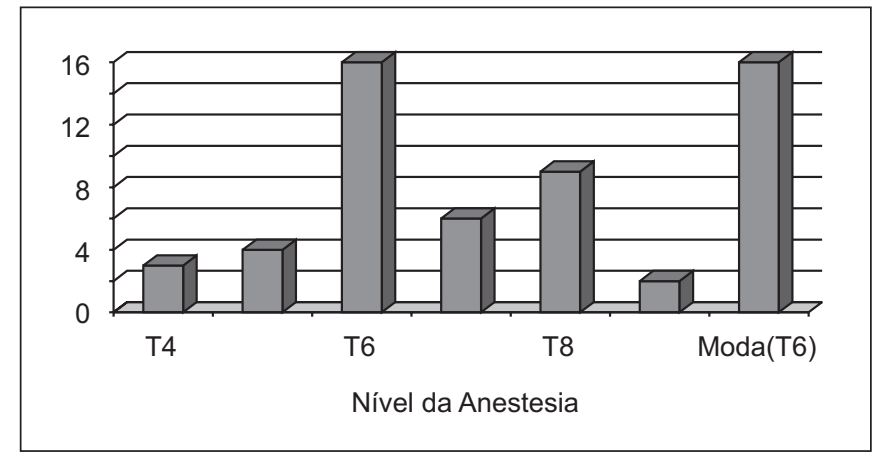

Figura 1 - Dispersão Cefálica da Analgesia

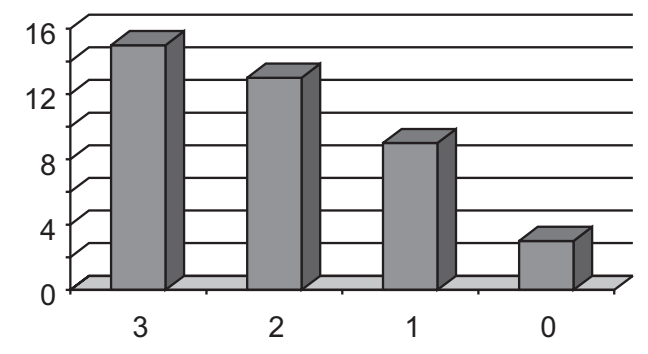

Graus de Bloqueio Motor (Bromage)

Figura 2 - Graus de Bloqueio Motor no Final da Cirurgia

Não ocorreu dessaturação nem hipotensão arterial que necessitasse de tratamento com vasopressor. Pelo teste de Kruskal-Wallis $(p<0,05)$ houve diferença significativa na PAS entre os valores PPR-IC e TC-PPR; na PAD entre IC-SO e TC-SO e na PAM entre PPR-SO, assim como na FC entre os valores PPR-SO, TC-SO e TC-IC (Tabela IV). Houve uma correlação da diminuição da freqüência cardíaca com o nível mais alto de bloqueio atingido. Bradicardia foi observada em dois pacientes, corrigida pela atropina. Ocorreu uma falha, sendo a cirurgia realizada sob anestesia geral. Vômito ocorreu em uma criança. Não foi observada cefaléia nem SNT.

Tabela IV - Avaliação Hemodinâmica nos Diferentes Momentos

\begin{tabular}{lcccccccc}
\hline & \multicolumn{2}{c}{ PAS $(\mathrm{mmHg})$} & \multicolumn{2}{c}{ PAM $(\mathrm{mmHg})$} & \multicolumn{2}{c}{ PAD $(\mathrm{mmHg})$} & \multicolumn{2}{c}{$\mathrm{FC}(\mathrm{bpm})$} \\
\cline { 2 - 8 } & $\mathrm{M} \pm \mathrm{DP}$ & Mediana & $\mathrm{M} \pm \mathrm{DP}$ & Mediana & $\mathrm{M} \pm \mathrm{DP}$ & Mediana & $\mathrm{M} \pm \mathrm{DP}$ & $\mathrm{Mediana}$ \\
\hline Chegada (SO) & $107 \pm 16$ & 102,00 & $67 \pm 15$ & 70,00 & $67 \pm 11$ & 67,00 & $95 \pm 10$ & 95,50 \\
Pós-punção (PPR) & $97 \pm 10$ & 98,00 & $64 \pm 11$ & 65,00 & $62 \pm 14$ & 63,00 & $03 \pm 15$ & 94,00 \\
Incisão (IC) & $103 \pm 15$ & 101,00 & $65 \pm 15$ & 65,00 & $64 \pm 12$ & 62,00 & $95 \pm 12$ & 99,00 \\
Término (TC) & $106 \pm 14$ & 103,00 & $63 \pm 15$ & 61,00 & $61 \pm 14$ & 59,00 & $90 \pm 11$ & 92,00 \\
\hline
\end{tabular}




\section{DISCUSSÃO}

No presente estudo, a raquianestesia com bupivacaína combinada com anestesia inalatória sob máscara para punção subaracnóidea produz uma anestesia segura para cirurgias ambulatoriais em crianças com idades entre 6 e 12 anos. Ocorreu falha em um paciente em que foi complementado com anestesia geral.

A anestesia inalatória superficial ou sedação torna difícil a avaliação do bloqueio sensitivo em crianças pequenas. Assim que a punção foi realizada, o anestésico inalatório era descontinuado e a pesquisa do nível sensitivo por picada da agulha foi possível em quase todos os pacientes.

Em recém-nascidos e crianças a dose de bupivacaína varia de 0,2 a $1 \mathrm{mg} \cdot \mathrm{kg}^{-1}$ em diversos trabalhos publicados. Em crianças de 2 a 115 meses a dose de $0,4 \mathrm{mg} \cdot \mathrm{kg}^{-1}$ de bupivacaína a $0,5 \%$ isobárica e hiperbárica proporcionou bloqueio sensitivo mediano em $\mathrm{T}_{4}{ }^{11}$, desnecessário para cirurgias na região inguinal e perineal. Com a mistura enantiomérica de bupivacaína (S75-R25) na dose de $0,5 \mathrm{mg} \cdot \mathrm{kg}^{-1}$ em crianças de 1 a 5 anos o nível médio obtido foi $\mathrm{T}_{6}{ }^{8}$. Neste trabalho utilizamos a dose de $0,5 \mathrm{mg} \cdot \mathrm{kg}^{-1}$ de bupivacaína em crianças de 6 a 12 anos e o nível mediano de bloqueio sensitivo obtido foi o mesmo $\left(T_{6}\right)$. Apesar da ampla variação na dose, a bupivacaína apresentou nível e duração adequados para quase todos os procedimentos indicados. Neste estudo, apenas uma criança necessitou complementação com anestesia geral. Sedação ou anestesia geral são freqüentemente necessárias para a realização de bloqueios em crianças. O óxido nitroso pode ser administrado para a realização do bloqueio ou mesmo infiltração da pele com anestésico local. Alguns autores contra-indicam uma sedação profunda pela possibilidade de parestesia durante a punção subaracnóidea, aumentando o risco de lesão neurológica permanente ${ }^{12}$. Neste estudo, preferimos a indução inalatória com sevoflurano, pois era a rotina do serviço e não foi observado nenhum caso de complicação neurológica até 30 dias de acompanhamento.

$\mathrm{O}$ início do bloqueio com bupivacaína isobárica ${ }^{13}$ e mistura enantiomérica de bupivacaína isobárica ${ }^{8}$ em crianças é rápido, sendo menor que dois minutos. Neste estudo, com a bupivacaína, o tempo do início do bloqueio foi menor do que $3 \mathrm{mi}-$ nutos. É óbvio que a monitorização do nível do bloqueio em crianças é muito difícil. Em adultos foi observado um tempo de latência $(1,86 \mathrm{~min}){ }^{14}$ menor do que o obtido com as crianças de 1 a 5 anos $^{8}$ e também menor do que com crianças de 6 a 12 anos. Este fato pode ser explicado pela presença residual da anestesia geral, dificultando a resposta aos estímulos dolorosos. O início do bloqueio sensitivo com a bupivacaína na raquianestesia em crianças necessita de posteriores estudos.

Aincidência de efeitos colaterais foi muito baixa. Em apenas duas crianças foi necessária a utilização de atropina para correção da bradicardia. Diferente dos adolescentes e adultos, as crianças pequenas toleram altos níveis de bloqueio torácico com apenas discretas alterações cardiocirculatórias. Estudando os efeitos da raquianestesia em crianças com nível de bloqueio alto (torácico) não se observou a esperada diminuição da freqüência cardíaca e da pressão arterial comum com a simpatectomia causada ${ }^{15}$. Estudo mostrou que em crianças com menos de cinco anos ocorreu pequena ou nenhuma alteração na pressão arterial e na freqüência cardíaca após raquianestesia com nível sensitivo entre $T_{5}-T_{3}$, enquanto que crianças acima de seis anos podem apresentar uma variação da pressão arterial de 20 a 40\%, semelhante ao adulto ${ }^{16}$. Neste estudo, com crianças de 6 a 12 anos, foi observada diminuição das pressões arteriais sistólicas, diastólicas e médias nos três momentos avaliados, em comparação com a chegada à sala de operações. Entretanto, nenhuma necessitou de vasopressor para correção da pressão arterial.

Da mesma forma que com a lidocaína a $5 \%{ }^{3}$ ocorreu uma rápida recuperação do bloqueio motor instalado, sendo que $30 \%$ dos pacientes apresentavam bloqueio motor grau 1 e zero no final da cirurgia. Entretanto, a recuperação do bloqueio motor com a bupivacaína $(2,50 \mathrm{~h})$ em crianças de 6 a 12 anos mostrou-se maior ( 1,3 vezes) que com a mistura enantiomérica de bupivacaína $(1,89 \mathrm{~h})$ em crianças de 1 a 5 anos $^{8}$.

A raquianestesia pode ser associada a diversos efeitos adversos, sendo cefaléia, dor nas costas e sintomas neurológicos os mais comuns. A agulha utilizada foi a 26G Atraucan, que alia a ponta cortante com a ponta de lápis. Todos os tipos de agulhas de raquianestesia já foram utilizados em crianças. Neste estudo, diferentemente de outros, não foi observada cefaléia.

Em crianças, a cefaléia é freqüentemente moderada e de curta duração ${ }^{9,11}$. É razoável esperar a redução na incidência e gravidade da cefaléia pós-raquianestesia quando se utiliza agulha de fino calibre ${ }^{17}$. Neste estudo, utilizando aguIha $26 \mathrm{G}$ Atraucan não foi observado nenhum caso de cefaléia, da mesma forma quando se utilizou a mesma agulha em crianças de 1 a 5 anos $^{8}$. Contudo, estudos posteriores com grandes populações são necessários para avaliar a incidência e gravidade da cefaléia em crianças.

A falha observada neste trabalho foi associada à dificuldade de obtenção de LCR, da mesma forma que em outros estudos ${ }^{7,8}$.

Náuseas e vômitos são comuns em crianças submetidas à cirurgia ${ }^{18,19}$. No presente estudo, a raquianestesia foi associada à baixa incidência de vômito $(2,5 \%)$, um pouco maior do que $1 \%$ de outro estudo ${ }^{20}$.

A bupivacaína a $0,5 \%$ isobárica produz anestesia segura e efetiva em pacientes de 6 a 12 anos em regime ambulatorial. $O$ acesso ao espaço subaracnóideo é fácil e a técnica pode ser ensinada a todos os anestesiologistas. A raquianestesia proporcionou um alto grau de sucesso, bloqueio motor de duração intermediária, baixa incidência de efeitos colaterais, sem aumentar o tempo na SRPA. Cefaléia pós-punção parece ser rara em pacientes abaixo de 12 anos quando se utiliza agulha de fino calibre. Por suas vantagens, simplicidade, facilidade, economia, acreditamos que a raquianestesia deve ser mais difundida para pacientes pediátricos.

\section{AGRADECIMENTO}

Agradecemos ao Prof. José Antonio Cordeiro pela valiosa orientação no estudo estatístico. 


\section{Isobaric 0.5\% Bupivacaine for Spinal Anesthesia in Pediatric Outpatient Surgery of 6 to 12 Year Old Children. A Prospective Study}

Luiz Eduardo Imbelloni, TSA, M.D., Eneida Maria Vieira, M.D., Lúcia Beato, TSA, M.D., Carlos Zapatta, M.D.

\section{INTRODUCTION}

The first pediatric spinal anesthesia was reported in $1901^{1}$, but the technique remained unpopular until $1980^{2,3}$ when it was reintroduced as an alternative to general anesthesia in high-risk neonates ${ }^{4-6}$ and became popular for children between 2 months and 12 years of age ${ }^{5-7}$. Spinal anesthesia was not used in the Hospital de Base until May 2001, when it became a routine for outpatient procedures in children aged 1 to 5 years ${ }^{8}$. It has a fast onset, with uniform analgesia spread and excellent muscle relaxation.

Amide-type local anesthetics (bupivacaine and lidocaine) are regularly used for pediatric spinal anesthesia in low doses and with low systemic toxicity risk. Lidocaine action may be short lasting for some outpatient procedures in children ${ }^{3,9}$. Bupivacaine has a longer action as compared to lidocaine. Local anesthetics' baricity is a major factor influencing drug's spread in the CSF ${ }^{10}$. Hyperbaric ${ }^{2,3,11}$ and isobaric ${ }^{8,11}$ solutions are used in pediatric spinal anesthesia with excellent results.

This study aimed at evaluating clinical characteristics of $0.5 \%$ isobaric bupivacaine for spinal anesthesia in pediatric surgeries. Analgesia spread and regression, PACU stay and hospital discharge was evaluated.

\section{METHODS}

After the Hospitals' Ethical Committees approval, participated in this 2-month study 40 patients aged 6 to 12 years scheduled for outpatient pediatric procedures under spinal anesthesia with $0.5 \mathrm{mg} \cdot \mathrm{kg}^{-1}$ bupivacaine (Table I). Parents and older children were informed about the study and gave their informed consent. Patients with spinal anesthesia counterindications, such as intracranial pressure increase, hemorrhagic or neurological problems or infection at puncture site were excluded from the study.

Children were told not to eat solid food or drink milk for 6 to 8 hours before anesthesia. Information about fasting was also given to parents. Clear fluids could be ingested up to 2 hours before anesthetic induction. All children were premedicated with oral midazolam $\left(0.8 \mathrm{mg} \cdot \mathrm{kg}^{-1}\right), 20$ minutes before arriving at the OR. Anesthesia was induced under mask with oxygen and sevoflurane up to $5 \%$, followed by venous puncture and $0.9 \%$ sodium chloride infusion. After induction, children were placed in the left lateral position and lumbar puncture was performed at $L_{3}-L_{4}$ or $L_{4}-L_{5}$ interspace with disposable pediatric needles (26G Atraucan ${ }^{\circledR}$ - B. Braun Melsungen AG) without introducer, by the paramedian route. Correct needle po406
Table I - Surgical Procedures

\begin{tabular}{ll}
\hline Cryptorchidism & 1 \\
Ureteral fistula & 2 \\
Unilateral inguinal herniorrhaphy & 9 \\
Bilateral inguinal herniorrhaphy & 7 \\
Inguinal herniorrhaphy + Posttectomy & 2 \\
Umbilical + Inguinal Herniorrhaphy & 2 \\
Inguinal herniorrhaphy + Orchidopexia & 1 \\
Inguinal herniorrhaphy + Hydrocelectomy & 1 \\
Hydrocelectomy & 2 \\
Hypospadias & 1 \\
Orchidopexia & 4 \\
Postectomy & 6 \\
Postectomy + Hydrocelectomy & 2 \\
\hline
\end{tabular}

sitioning was confirmed by the presence of CSF. Bevel was placed in the cephalad direction and the calculated dose of $0.5 \%$ isobaric bupivacaine in sterilized package was injected without barbotage at the speed of $1 \mathrm{ml} .15 \mathrm{~s}^{-1}$. Patients were then immediately placed in the supine position. Perioperative monitoring consisted of non-invasive blood pressure at 5-minute intervals, heart rate and pulse oximetry $\left(\mathrm{SpO}_{2}\right)$. ECG was continuously monitored at CM5. When blood pressure decreased above $30 \%$ of baseline values, the initial treatment consisted in increasing crystalloids infusion and then ephedrine. Heart rate was considered normal when around $100 \mathrm{bpm}$. If there was a $15 \%$ decrease, $0.01 \mathrm{mg} \cdot \mathrm{kg}^{-1}$ atropine was administered. SBP, DBP, MBP and HR values were analyzed and compared in the following moments: OR arrival, post-dural puncture (PDP), surgical incision (SI) and surgery completion (SC).

After blockade and sevoflurane withdrawal, blockade onset was evaluated and defined as time for the first loss of sensitivity in the region corresponding to the puncture metamer. Segmental level of analgesia was defined as loss of pinprick sensation and was tested 10 minutes after anesthetic injection. Motor block was evaluated by Bromage's modified scale ( $0=$ no blockade and $3=$ total motor block $)$ in the beginning and the end of surgery.

Children were transferred to PACU after surgery for vital signs continuous monitoring and blockade regression evaluation. Analgesia duration was defined as the return of sensitivity on the dermatome corresponding to the puncture, and motor block duration was defined as total muscle activity recovery in lower limbs. All adverse effects were recorded. Pain was treated with intravenous $20 \mathrm{mg} \cdot \mathrm{kg}^{-1}$ dipirone. As soon as children would freely move their lower limbs, they were transferred to the outpatient unit.

Hospital discharge was authorized when children were awaken, ambulating normally with stable vital signs, no pain, no nausea or vomiting and were able to tolerate fluids and urinate spontaneously. Patients were followed until the third posVol. 52, N 4, Julho - Agosto, 2002 
toperative day for post-dural puncture headache or transient neurological symptoms (TNS) defined as back, buttocks or legs pain or dysesthesia, irradiating to lower extremities and resolving within 72 hours or up to 30 days for permanent neurological complications.

Results are shown as the mean \pm standard deviation and were analyzed by Kruskal-Wallis and Wilcoxon Signed Rank (median test) tests to compare blood pressure, heart rate and oxygen saturation, being considered significant when $p<$ 0.05 .

\section{RESULTS}

Demographics data is shown in table II.

Table II -Demographics Data

\begin{tabular}{lc}
\hline Age $(\text { years })^{*}$ & $8.15 \pm 1.95$ \\
Weight $(\mathrm{kg})^{*}$ & $31.98 \pm 11.05$ \\
Height $(\mathrm{cm})^{*}$ & $126.65 \pm 14.12$ \\
Gender & \\
$\quad$ Male & 29 \\
Female & 11 \\
\hline
\end{tabular}

*Values expressed in Mean \pm SD

Blockade characteristics are shown in table III. Onset time was $2.60 \pm 2.33 \mathrm{~min}$. Analgesia duration was $4.51 \pm 0.89 \mathrm{~h}$. Time for ambulation was $4.04 \pm 0.83 \mathrm{~h}$. PACU stay was 44.39 $\pm 43.13 \mathrm{~min}$. Motor block duration was $2.50 \pm 0.83 \mathrm{~h}$.

Table III - Blockade Characteristics (Mean \pm SD)

\begin{tabular}{lc}
\hline Onset (min) & $2.60 \pm 1.28$ \\
Analgesia (h) & $4.51 \pm 0.89$ \\
Motor block duration (h) & $2.50 \pm 0.83$ \\
Time for spinal anesthesia-ambulation (h) & $4.04 \pm 0.61$ \\
PACU stay (min) & $44.39 \pm 43.13$ \\
\hline
\end{tabular}

Sensory block level varied from $T_{9}$ to $T_{4}$ with Mode $=T_{6}$ (Figure 1).

Motor block onset was less than three minutes in all children. All of them presented motor block grade 3 at the beginning of the surgery. At the end of surgery, motor block grade 3 was observed in 15 patients, grade 2 in 13, grade 1 in 9 and grade 0 in 3 . Over $70 \%$ of all patients recovered to motor block 3 or 2 at surgery completion (Figure 2).

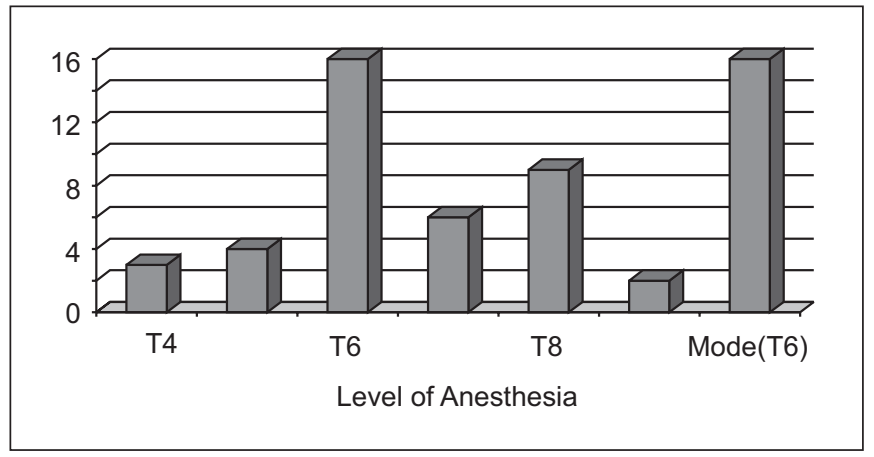

Figure 1 - Cephalad Spread of Analgesia

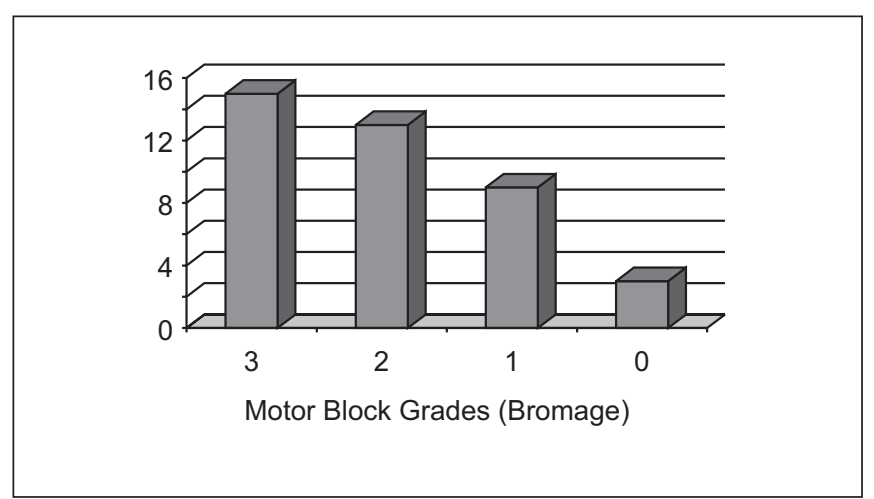

Figure 2 - Motor Block Grades at Surgery Completion

No patient showed oxygen desaturation or arterial hypotension needing treatment with vasopressor. Kruskall-Wallis test $(p<0.05)$ has shown a significant SBP difference between PPR-IC and TC-PPR; in DBP between IC-SO and TC-SO and in MBP between PPR-SO, as well as in HR between PPR-SO, TC-SO and TC-IC (Table IV). There has been a correlation between heart rate decrease and highest motor block level. Bradycardia was observed in two patients and corrected with atropine. There has been one motor block failure and surgery was performed under general anesthesia. Vomiting was observed in one child. There were no PDPH or transient neurological symptoms.

Table IV - Hemodynamic Evaluation in Different Moments

\begin{tabular}{|c|c|c|c|c|c|c|c|c|}
\hline & \multicolumn{2}{|c|}{$\mathrm{SBP}(\mathrm{mmHg})$} & \multicolumn{2}{|c|}{$\mathrm{MBP}(\mathrm{mmHg})$} & \multicolumn{2}{|c|}{$\mathrm{DBP}(\mathrm{mmHg})$} & \multicolumn{2}{|c|}{ HR (bpm) } \\
\hline & $M \pm D P$ & Median & $M \pm D P$ & Median & $M \pm D P$ & Median & $M \pm D P$ & Median \\
\hline Arrival (OR) & $107 \pm 16$ & 102.00 & $67 \pm 15$ & 70.00 & $67 \pm 11$ & 67.00 & $95 \pm 10$ & 95.50 \\
\hline Incision (SI) & $103 \pm 15$ & 101.00 & $65 \pm 15$ & 65.00 & $64 \pm 12$ & 62.00 & $95 \pm 12$ & 99.00 \\
\hline Completion (SC) & $106 \pm 14$ & 103.00 & $63 \pm 15$ & 61.00 & $61 \pm 14$ & 59.00 & $90 \pm 11$ & 92.00 \\
\hline
\end{tabular}




\section{DISCUSSION}

In our study, spinal anesthesia with bupivacaine combined with inhalational anesthesia under mask for spinal puncture has induced a safe anesthesia for outpatient procedures in children aged 6 to 12 years. There has been one failure and surgery was performed under general anesthesia.

Superficial inhalational anesthesia or sedation impairs sensory block evaluation in small children. Soon after puncture, inhalational anesthesia was discontinued and sensory block evaluation by pinprick was possible in almost all patients.

In neonates and small children, bupivacaine dose varies from 0.2 to $1 \mathrm{mg}^{\mathrm{kg}}{ }^{-1}$ in several studies. In children between 2 and 115 months of age, $0.4 \mathrm{mg}^{\mathrm{kg}} \mathrm{kg}^{-1}$ of $5 \%$ isobaric and hyperbaric bupivacaine has induced a median sensory block in $\mathrm{T}_{4}$ ${ }^{11}$, unnecessary for inguinal and perineal surgeries. With 0.5 $\mathrm{mg} . \mathrm{kg}^{-1}$ isobaric enantiomeric mixture of bupivacaine (S75-R25) in children aged 1 to 5 years, the mean level obtained was $\mathrm{T}_{6}{ }^{8}$. In our study, we have used $0.5 \mathrm{mg} \cdot \mathrm{kg}^{-1}$ bupivacaine in children aged 6 to 12 years and median sensory block level was the same $\left(T_{6}\right)$. In spite of wide dose variations, bupivacaine induced an adequate level and duration for almost all procedures. In our study, only one child needed complementation with general anesthesia.

Sedation or general anesthesia are often needed for pediatric blockade. Nitrous oxide may be administered for blockade or even skin infiltration with local anesthetics. Some authors counterindicate deep sedation due to the risk for paresthesia during spinal puncture, thus increasing the risk for permanent neurological injuries ${ }^{12}$. In our study, we decided for inhalational induction with sevoflurane because this is our routine and no neurological complications were seen after 30 days of follow-up.

Blockade onset with isobaric bupivacaine ${ }^{13}$ and isobaric enantiomeric mixture of bupivacaine ${ }^{8}$ in children is fast and takes less than two minutes. In our study with bupivacaine, blockade onset was less than three minutes. It is clear that blockade level monitoring is very difficult in children. Adults have shorter onset times $(1.86 \mathrm{~min})^{14}$ as compared to 1 to 5 -year old children ${ }^{8}$ and to 6 to 12 -year old children. This may be explained by the presence of residual general anesthesia which impairs the response to painful stimulations. Sensory block onset with bupivacaine for pediatric spinal anesthesia requires further studies.

There has been a low incidence of side-effects. Only two children needed atropine to correct bradycardia. Different from adolescents and adults, small children tolerate high thoracic block levels with just mild cardiocirculatory changes. Studying the effects of spinal anesthesia in children with high blockade levels (thoracic), the expected decrease in heart rate and blood pressure, associated to sympathecthomy, was not observed ${ }^{15}$. A study has shown that in children below 5 years of age there has been a mild or no change in blood pressure and heart rate after spinal anesthesia with sensory block level between $T_{5}-T_{3}$, while children above 6 years of age may have blood pressure variations of $20 \%$ to $40 \%$, similar to adults ${ }^{16}$. In our study with children aged 6 to 12 years, systo- lic, diastolic and mean blood pressure decreased in the three evaluated moments, as compared to OR arrival, however, none of them needed vasopressors to correct blood pressure.

Similarly to $5 \%$ lidocaine ${ }^{3}$, there is a fast motor block recovery and $30 \%$ of patients presented motor block grade one and zero at surgery completion. However, motor block recovery with bupivacaine $(2.50 \mathrm{~h})$ in children aged 6 to 12 years was longer (1.3 times) as compared to enantiomeric mixture of bupivacaine $(1.89 \mathrm{~h})$ in children aged 1 to 5 years $^{8}$.

Spinal anesthesia may be associated to several adverse effects, the most common being headache, back pain and neurological symptoms. 26G Atraucan was the needle of choice for allying a cutting edge and a pencil point. All types of spinal anesthesia needles have already been used in children. In our study, and differently from others, there has been no headache.

Headache in children is in general moderate and brief ${ }^{9,11}$. it is reasonable to expect a decrease in post-dural puncture headache incidence and severity when a fine needle is used ${ }^{17}$. In our study with 26G Atraucan needles, no headache was seen, similarly to what has been observed when the same needle was used in children between 1 and 5 years of age ${ }^{8}$. However, further studies with larger samples are needed to evaluate headache incidence and severity in children.

The failure observed in our study was associated to difficulty in obtaining CSF, and was in line with other studies ${ }^{7,8}$. Nausea and vomiting are common in children submitted to surgical procedures ${ }^{18,19}$. In our study, spinal anesthesia was associated to a low incidence of vomiting $(2.5 \%)$, slightly higher than the $1 \%$ found by a different study ${ }^{20}$.

Isobaric $0.5 \%$ bupivacaine induces a safe and effective anesthesia for outpatient procedures in patients aged 6 to 12 years. Spinal space access is easy and the technique may be taught to all anesthesiologists. Spinal anesthesia has provided a high success rate, with short-lasting motor block, low incidence of side-effects and without increasing PACU stay. Post-dural puncture headache seems to be rare in patients below 12 years of age when a fine needle is used. For its advantages, simplicity, easiness and low cost, we believe that spinal anesthesia should be more popular for pediatric patients.

\section{ACKNOWLEDGEMENT}

We acknowledge Prof. José Antonio Cordeiro for his invaluable advice during statistical analysis.

\section{REFERÊNCIAS - REFERENCES}

01. Bainbridge WS - A report of twelve operations on infants and young children during spinal analgesia. Arch Pediatr, 1901;18: 510-520.

02. Berkowitz S, Greene BA - Spinal anesthesia in children: Report based on 350 patients under 13 years of age. Anesthesiology, 1951;12:376-387 
03. Gouveia MA - Raquianestesia em pacientes pediátricos. Experiência pessoal em 50 casos. Rev Bras Anestesiol, 1970; 20:503-511.

04. Abajian JC, Mellish RWP, Browne AF et al - Spinal anesthesia for surgery in the high-risk infant. Anesth Analg, 1984;63:359362.

05. Harnik EV, Hoy GR, Potolicchio S et al - Spinal anesthesia in premature infants recovering from respiratory distress syndrome. Anesthesiology, 1986;64:95-99.

06. Veverka TJ, Henry DN, Milroy LN et al - Spinal anesthesia reduces the hazard of apnea in high-risk infants. Am Surg, 1991; 57:531-534.

07 . Kokki $\mathrm{H}$, Hendolin $\mathrm{H}$ - No difference between bupivacaína in $0.9 \%$ and $8 \%$ glucose for spinal anaesthesia in small children. Acta Anaesthesiol Scand, 2000;44:548-551.

08. Imbelloni LE, Vieira EM, Beato L et al - Mistura enantiomérica de bupivacaína (S75:R25) isobárica para raquianestesia em crianças de 1 a 5 anos em cirurgia pediátrica ambulatorial. Rev Bras Anestesiol, 2002,52:286-293.

09. Kokki H, Hendolin H - Comparison of 25G and 29G Quincke spinal needles in paediatric day case surgery. A prospective randomized study of the puncture characteristics, success, rate and postoperative complaints. Paediatr Anaesth, 1996;6: 115-119.

10. Imbelloni LE - O Uso Racional da Raquianestesia, em: Imbelloni LE - Tratado de Anestesia Raquidiana, Curitiba, 2001;8

11. Kokki H, Tuovinen K, Hendolin H - Spinal anaesthesia for paediatric day-case surgery: a double-blind randomized parallel group, prospective comparison of isobaric and hyperbaric bupivacaine. Br J Anaesth, 1998;81:502-506.

12. Horlocker TT, McGregor DB, Matsushinge DK et al - A retrospective review of 4767 consecutive spinal anesthetics: central nervous system complications. Anesth Analg, 1997;84:578-584.

13. Mahe V, Ecoffey $C$ - Spinal anesthesia with isobaric bupivacaine in infants. Anesthesiology, 1988;68:601-603.

14. Imbelloni LE, Beato L - Comparação entre bupivacaína racêmica (S50:R50) e mistura enantiomérica de bupivacaína (S75:R25), ambas isobáricas, a $0,5 \%$ em raquianestesia. Estudo em cirurgias ortopédicas. Rev Bras Anestesiol, 2001; 51:369-376.

15. Oberlander TF, Berde CB, Lam KH et al - Infants tolerate spinal anaesthesia with minimal overall autonomic changes: analysis of heart rate variability in former premature infants undergoing hernia repair. Anesth Analg, 1995;80:20-27.

16. Dohi S, Naito H, Takahashi T - Age-related changes in blood pressure and duration of motor block in spinal anesthesia. Anesthesiology, 1979;50:319-323.

17. Imbelloni LE, Sobral MGC, Carneiro ANG - Cefaléia pósraquianestesia e o desenho das agulhas. Experiência com 5050 casos. Rev Bras Anestesiol, 2001;51:43-52.
18. Karlsson E, Larsson LE, Nilsson K - Postanaesthetic nausea in children. Acta Anaesthesiol Scand, 1990;34:515-518.

19. Kokki H, Ahonen R - Pain and activity disturbance after paediatric day case adenoidectomy. Paediatr Anaesth, 1997;7: 227231.

20. Kokki H, Heikkinen M, Ahonen R - Recovery after paediatric daycase herniotomy performed under spinal anaesthesia. Paediatr Anaesth, 2000;10:413-417.

\section{RESUMEN}

Imbelloni LE, Vieira EM, Beato L, Zapatta C - Raquianestesia con Bupivacaína a $0,5 \%$ Isobárica para Cirugía Pediátrica Ambulatorial en Pacientes con Edades de 6 a 12 años. Estudio Prospectivo

Justificativa y Objetivos - En niños, la raquianestesia produce profunda analgesia y, cuando combinada con anestesia general, reduce la necesidad de los agentes anestésicos y de los opioides. El objetivo de este estudio prospectivo fue evaluar las características clínicas de la raquianestesia con bupivacaína a $0,5 \%$ racémica en 40 niños, con edades entre 6 e 12 años.

Método - Participaron del estudio prospectivo 40 niños con edades entre seis y 12 años, sometidos a raquianestesia con bupivacaína a $0,5 \%$ isobárica, en la dosis de $0,5 \mathrm{mg} \cdot \mathrm{kg}^{-1}$. Fueron evaluados los siguientes parámetros: latencia de la analgesia, bloqueo motor, duración de los efectos, dispersión craneal de la analgesia, alteraciones cardiovasculares, cefalea, síntomas neurológicos transitorios.

Resultados - El tiempo de latencia fue de 2,60 $\pm 1,28$ minutos. La duración de la analgesia fue de 4,51 $\pm 0,89 \mathrm{~h}$. El tiempo para la deambulación fue de 4,04 $\pm 0,83 \mathrm{~h}$. El tiempo de permanencia en la SRPA fue de 44,39 \pm 43,13 minutos. La duración del bloqueo motor fue de 2,50 $\pm 0,83 \mathrm{~h}$. El nivel sensitivo del bloqueo varió entre $T_{9}$ y $T_{4}\left(M o d a=T_{6}\right)$. El inicio del bloqueo motor fue menor que tres minutos en todos los niños. Luego en el inicio de la cirugía, todos los pacientes presentaron bloqueo motor grado 3 (escala modificada de Bromage). Setenta por ciento de los pacientes presentaron bloqueo motor grados 3 ó 2 en el final de la cirugía. No fue observada desaturación o hipotensión arterial. Bradicardia fue observada en dos pacientes. Ocurrió una falla. Cefalea y SNT no fueron observadas.

Conclusiones - En las condiciones de este estudio la bupivacaína a 0,5\% isobárica produjo anestesia segura en pacientes de 6 a 12 años en régimen ambulatorial, con alto índice de suceso, bloqueo motor de duración intermediaria y baja incidencia de efectos colaterales. Los resultados mostraron que la raquianestesia es segura y fácilmente realizable en niños de 6 a 12 años en régimen ambulatorial. 